\title{
Galaxy spectra from the UV to the mid-IR
}

\author{
Michael J. I. Brown ${ }^{1}$, John Moustakas ${ }^{2}$, J.-D. T. Smith ${ }^{3}$, Elisabete da \\ Cunha $^{4}$, Masatoshi Imanishi ${ }^{5,6}$, Lee Armus ${ }^{7}$, and Bernhard R. Brandl ${ }^{8}$ \\ ${ }^{1}$ School of Physics, Monash University, Clayton, Victoria 3800, Australia \\ email: Michael.Brown@monash.edu \\ ${ }^{2}$ Department of Physics and Astronomy, Siena College, 515 Loudon Road, Loudonville, NY \\ 12211-1462, USA \\ ${ }^{3}$ Department of Physics and Astronomy, University of Toledo, Ritter Obs., MS \#113, Toledo, \\ $\mathrm{OH} 43606$, USA \\ ${ }^{4}$ Max Planck Institute for Astronomy, Königstuhl 17, 69117 Heidelberg, Germany \\ ${ }^{5}$ Subaru Telescope, 650 North A'ohoku Place, Hilo, HI 96720, USA \\ ${ }^{6}$ Department of Astronomy, School of Science, Graduate University for Advanced Studies \\ (Sokendai), Mitaka, Tokyo 181-8588, Japan \\ ${ }^{7}$ Spitzer Science Center, California Institute of Technology, Pasadena, CA 91125, USA \\ ${ }^{8}$ Leiden Observatory, Leiden University, P.O. Box 9513, 2300 RA Leiden, The Netherlands
}

\begin{abstract}
Templates and models of galaxy spectra are often essential for determining the physical properties of galaxies. Many commonly used templates have large systematic errors, which significantly impact photometric redshifts and k-corrections. We present a new library of 110 galaxy template spectra spanning from the ultraviolet to the mid-infrared. The templates combine optical, Spitzer and Akari spectra with MAGPHYS models, all normalised and verified with matched aperture photometry. Our library contains more galaxies, spans a broader range of colours and has smaller systematic errors than previous libraries of galaxy spectra.
\end{abstract}

Keywords. galaxies: general, galaxies: fundamental parameters, galaxies: distances and redshifts, infrared: galaxies

\section{Introduction}

Libraries and models of galaxy spectra are often essential for determining rest-frame luminosities and photometric redshifts. The Coleman et al. (1980) and Kinney et al. (1996) templates have proved exceptionally useful over recent decades, but have limited wavelength coverage. With the advent of the Spitzer Space Telescope, Akari (Astro-F) and the Wide-field Infrared Space Explorer (WISE), it became both important and possible to extend galaxy spectral templates into the mid-infrared. Templates from Polletta et al. (2008) and others now include the emission from dust and polycyclic aromatic hydrocarbons (PAHs), but systematic errors remain that can overwhelm random errors when determining k-corrections and photometric redshifts.

Galaxy spectral energy distributions can be modelled, using models of stellar populations, nebular emission lines, dust obscuration and dust emission. The models have improved considerably over the decades, and in some instances do a remarkably good job of reproducing galaxy spectra. However, the models can have a large number of free parameters and cannot be expected to reproduce the spectral energy distribution of a galaxy with a complex star formation history when only limited photometry is available. This being the case, there is still a role for spectral templates derived from real galaxies. 

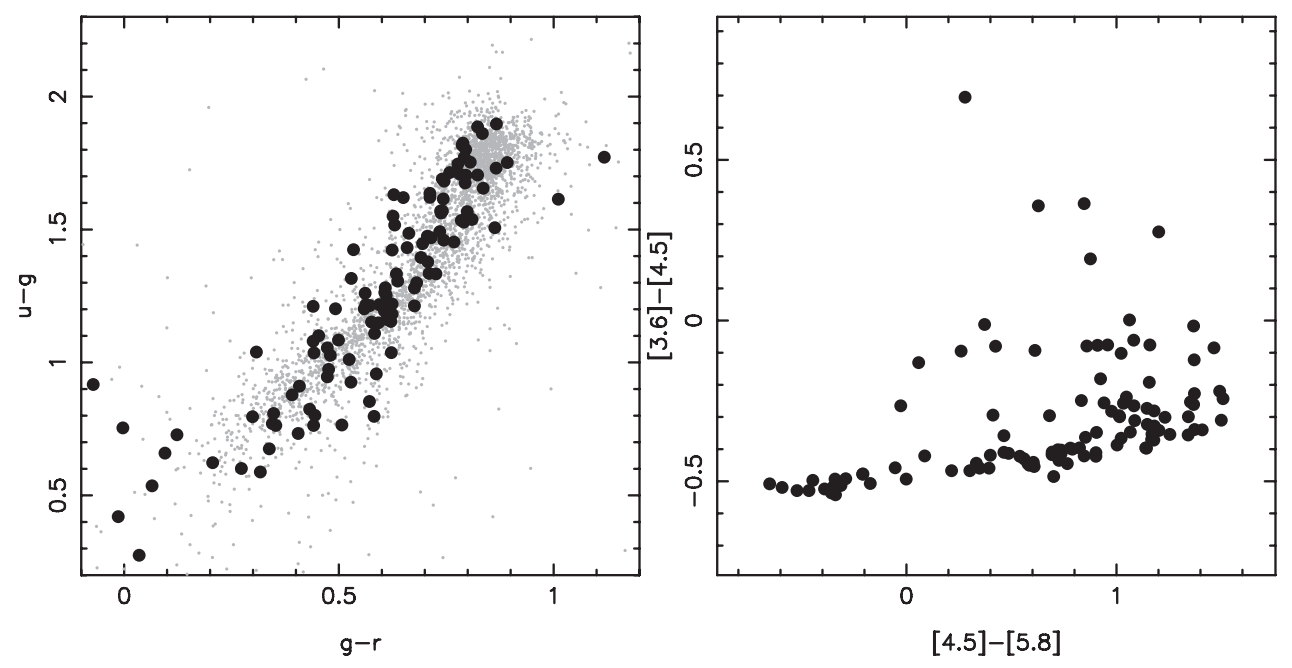

Figure 1. The optical and Spitzer infrared colours of the sample galaxies. While many galaxies do fall on a narrow galaxy locus, there are exceptions including star forming galaxies with strong nebular emission lines and some luminous infrared galaxies.

\section{A New Library of Galaxy Spectra}

We present a new library template spectra of galaxies spanning from the ultraviolet to the mid-infrared. Our sample consists of local galaxies that have drift-scan optical spectroscopy from Moustakas \& Kennicutt (2006), Moustakas et al. (2010), Kennicutt (1992) or Gavazzi et al. (2004). With the exception of a few elliptical galaxies, almost all of our galaxies also have spectra from Spitzer's Infrared Spectrograph (IRS). We make use of International Ultraviolet Explorer (IUE) and Akari Infrared Camera (IRC) spectroscopy when they capture a high fraction of the total flux and have high signal-tonoise. We used published spectra when possible (e.g., Smith et al. 2007, Imanishi et al. 2010), but for some galaxies we reduced data from the Spitzer and Akari archives.

Matched aperture photometry is critical for normalising and verifying our spectra. We make use of imaging from GALEX Data Release 6 (GR6), XMM-Newton optical/UV monitor telescope, Sloan Digital Sky Survey III (SDSS-III), Two Micron All Sky Survey (2MASS), Wide-field Infrared Survey Explorer (WISE) and Spitzer. The aperture is typically matched to the aperture used for the optical spectroscopy. Most galaxies are bright, so we expect systematic errors to dominate over random errors, but for the faintest galaxies we measure uncertainties using 24 sky apertures placed around the target galaxy.

We plot optical and infrared colour-colour diagrams for our galaxies in Figure 1. While many galaxies lie on a tight stellar locus, there are notable exceptions. Galaxies with strong nebular emission lines produce a wedge at the bottom-left of the optical colourcolour diagram. While the Rayleigh-Jeans tail dominates the spectra of most galaxies at $\sim 4 \mu \mathrm{m}$, some galaxies have strong absorption features and hot dust emission. Libraries with a limited number of spectra may not capture the true diversity of local galaxies.

We normalise and verify our spectra with the matched aperture photometry. For compact galaxies with stare mode spectra from Spitzer's IRS, we use the photometry to correct for the varying fraction of light that passes through the spectrograph slit as a function of wavelength. To fill the gaps in our spectral coverage, we use MAGPHYS (da Cunha, Charlot \& Elbaz 2008) models constrained with our photometry. As MAGPHYS does not include nebular emission lines, we correct our photometry for the contribution of emission lines (using our spectra) before fitting models to the data. In Figure 2 we 

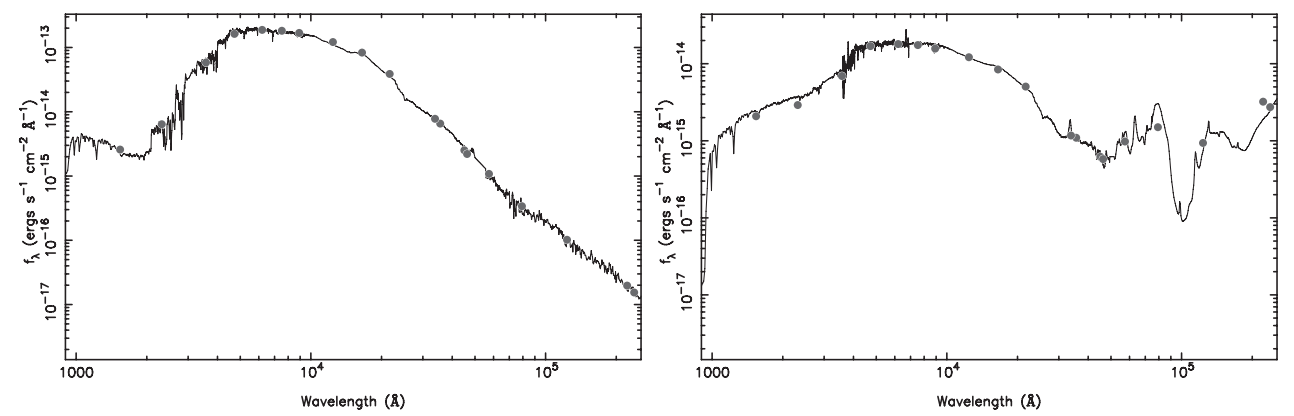

Figure 2. The spectral energy distributions of NGC 584 (left) and IC 4553 (Arp 220; right). The photometry used to normalise and verify the spectra are shown with the blue dots.
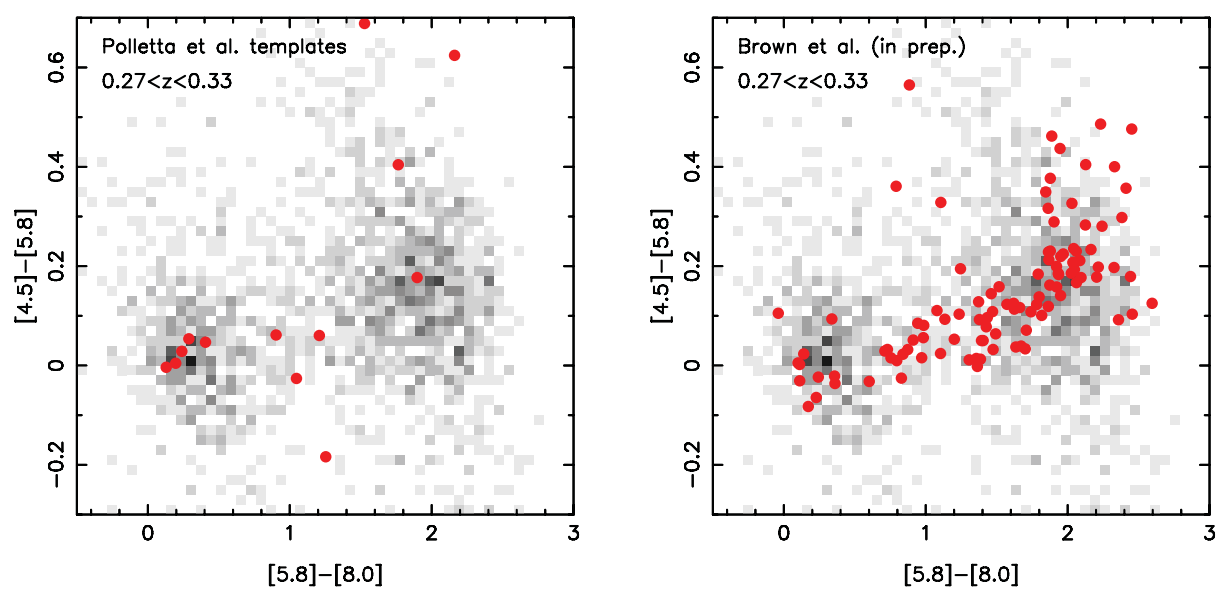

Figure 3. A comparison of infrared colours of $z_{\text {spec }} \sim 0.3$ galaxies with the templates of Polletta et al. (2008) (left panel) and our templates (right panel; Brown et al. in prep.). The loci of passive and star forming galaxies are evident to the left and right of each panel respectively. Our 110 template spectra clearly follow the observed galaxy locus and there are few outliers.

plot example spectra for NGC 584 and IC 4553 (Arp 220). While NGC 584 falls on the galaxy locus in the optical and infrared, IC 4553 has a redder than normal [3.6] - [4.5] colour due to ice absorption and hot dust emission (Imanishi et al. 2010).

In Figure 3 we compare our templates and the Polletta et al. (2008) templates with the observed infrared colours of $z_{\text {spec }} \sim 0.3$ galaxies in Bootes (Jannuzi \& Dey 1999, Kochanek et al. 2012). While several Polletta et al. (2008) templates populate the passive galaxy locus, only one template falls in the centre of the star forming galaxy locus. With over 100 templates, we define a very tight galaxy locus with only a small number of outliers. As our outliers have had their spectral energy distributions cross checked with matched aperture photometry, we are confident they represent real galaxies rather than errors in the spectra.

Our primary goal is to provide improved template spectral energy distributions for photometric redshifts and k-corrections. In Figure 4 we plot the rest-frame optical colourmagnitude diagrams for $z_{\text {spec }} \sim 0.5$ galaxies in Bootes determined using the Polletta et al. (2008) templates and our templates. To determine the rest-frame luminosities we used the method of Rudnick et al. (2003), where the spectral energy distributions are used to interpolate between two observed filters that bracket the relevant rest-frame filters. While this method reduces the impact of systematic errors on the rest-frame colours, we 

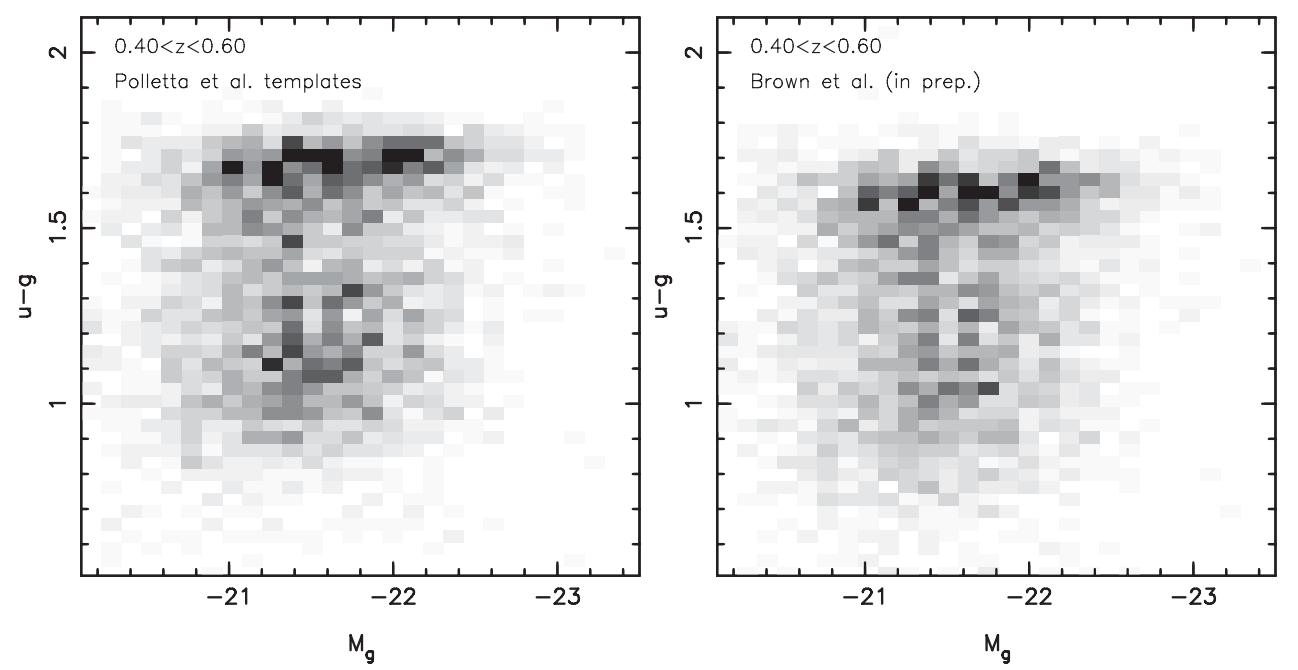

Figure 4. The rest-frame optical colour-magnitude diagrams of $z_{s p e c} \sim 0.5$ galaxies in the Bootes field. In the left panel the Polletta et al. (2008) templates were used to derive the rest-frame colours while in the right panel our templates were used. Despite using the method of Rudnick et al. (2003), which reduces the impact of systematic errors on k-corrections, we do observe significant differences when using different libraries of template spectra.

still see significant differences between the k-corrections derived from the two template libraries. Relative to the Polletta et al. (2008) templates, our templates shift the red sequence slightly blueward and produce a more dispersed locus of star forming galaxies.

\section{Summary}

Motivated by systematic errors in existing libraries of galaxy template spectra, we have developed a new library of 110 template spectra. The library contains more spectra, spans a broader wavelength range, has smaller uncertainties and has smaller systematic errors than comparable libraries. Preliminary tests indicate that the new library produces improved photometric redshifts and k-corrections. We find that galaxy k-corrections have a significant dependence on the libraries of template spectra used, even when applying methods that should mitigate the impact of systematic errors on the k-corrections.

\section{References}

Coleman, G. D., Wu, C.-C., \& Weedman, D. W. 1980, ApJS, 43, 393

da Cunha, E., Charlot, S., \& Elbaz, D. 2008, MNRAS, 388, 1595

Gavazzi, G., Zaccardo, A., Sanvito, G., Boselli, A., \& Bonfanti, C. 2004, A\& A, 417, 499

Imanishi, M., Nakagawa, T., Shirahata, M., Ohyama, Y., \& Onaka, T. 2010, ApJ, 721, 1233

Jannuzi, B. T. \& Dey, A. 1999, Photometric Redshifts and the Detection of High Redshift Galaxies, ASP Conference Series, 191, 111

Kennicutt, R. C., Jr. 1992, ApJS, 79, 255

Kinney, A. L., Calzetti, D., Bohlin, R. C., et al. 1996, ApJ, 467, 38

Kochanek, C. S., Eisenstein, D. J., Cool, R. J., et al. 2012, ApJS, 200, 8

Moustakas, J. \& Kennicutt, R. C., Jr. 2006, ApJS, 164, 81

Moustakas, J., Kennicutt, R. C., Jr., Tremonti, C. A., et al. 2010, ApJS, 190, 233

Polletta, M., Omont, A., Berta, S., et al. 2008, A\&A, 492, 81

Rudnick, G., Rix, H.-W., Franx, M., et al. 2003, ApJ, 599, 847

Smith, J. D. T., Draine, B. T., Dale, D. A., et al. 2007, ApJ, 656, 770 